\title{
Blood transfusion increases radical promoting non-transferrin bound iron in preterm infants
}

\author{
K Hirano, T Morinobu, H Kim, M Hiroi, R Ban, S Ogawa, H Ogihara, H Tamai, \\ T Ogihara
}

\begin{abstract}
Background-Blood transfusion has been recognised as a risk factor for the development of retinopathy of prematurity (ROP) or chronic lung disease (CLD) in preterm infants, but the precise mechanism involved is not understood.

Aim-To investigate the level of nontransferrin bound "free" iron, which has the potential to promote the generation of reactive oxygen species, and its redox status in the plasma of preterm infants immediately before and after blood transfusion.

Methods-Twenty one preterm infants with a median gestational age and birth weight of 27 weeks and $1021 \mathrm{~g}$ respectively were prospectively enrolled in the study. Sixteen of the 21 infants developed ROP and/or CLD. The infants were transfused with concentrated red blood cells at a median age of 32 days. The plasma concentration of total bleomycin detectable iron (BDI) was measured and also the ferrous iron $\left(\mathrm{Fe}^{2+}\right)$ activity by bleomyciniron complex dependent degradation of DNA.
\end{abstract}

Results-Even before blood transfusion, BDI was detectable in one third of the blood samples, and all but one sample had ferrous iron activity. After transfusion, both BDI and ferrous iron activity were significantly increased, in contrast with the situation in full term infants. Plasma ascorbic acid (AA) concentration was significantly decreased after blood transfusion, whereas the level of its oxidation product, dehydroascorbic acid (DHAA), and the DHAA/AA ratio were significantly increased compared with before the transfusion. The activity of plasma ferroxidase, which converts iron from the ferrous to the ferric state, was appreciably decreased in preterm infants, as expected from their very low plasma caeruloplasmin concentration.

Conclusions-Plasma non-transferrin bound iron was significantly increased in preterm infants after blood transfusion and existed partly in the ferrous form, because of the low ferroxidase activity and the reduction of ferric iron $\left(\mathrm{Fe}^{3+}\right)$ by ascorbic acid. This finding was specific to preterm infants and was not observed in full term infants after blood transfusion. Non-transferrin bound "free" iron may catalyse the generation of reactive oxygen species, which may be responsible for the clinical association of blood transfusion with ROP and CLD.

(Arch Dis Child Fetal Neonatal Ed 2001;84:F188-F193)

Keywords: retinopathy of prematurity; chronic lung disease; iron; ascorbic acid; blood transfusion; preterm

As preterm infants are prone to developing anaemia during the first few months of life because of rapid growth, insufficient erythropoiesis, and blood sampling for clinical purposes, blood transfusion is often necessary to improve their haemodynamics and respiratory status. Several studies have suggested that blood transfusion is a risk factor for retinopathy of prematurity $(\mathrm{ROP})^{1-3}$ and chronic lung disease (CLD), ${ }^{45}$ but the precise mechanism is not known. It has been reported that the mean lifespan of red blood cells transfused into preterm infants is much shorter than that of those transfused into adults. ${ }^{6}$ Therefore transfusion may lead to relative iron overload in preterm infants as the result of the accelerated breakdown of red blood cells.

It has been suggested that free radicals are involved in the pathogenesis of ROP, CLD, necrotising enterocolitis, and intracranial haemorrhage of prematurity..$^{7-9}$ Neonates, especially preterm infants, are thought to be at particular risk of oxidative stress because of incomplete maturation of the antioxidant defences. ${ }^{10-12}$ The presence of non-transferrin bound, bleomycin detectable iron (BDI) has been reported in the cord plasma of preterm infants and even uncomplicated full term infants. ${ }^{13}$ Cooke et $a l^{4}$ showed the gradual increase in serum BDI levels with age in preterm infants with CLD. BDI is a potentially redox active, powerful promoter of the formation of highly reactive oxygen species. ${ }^{14}$ For instance, in the presence of redox active iron, ascorbic acid (AA) can act as a pro-oxidant in vitro and contribute to the formation of hydroxyl radicals. ${ }^{15}$ The human body is normally very careful to sequestrate plasma iron into the safe transferrin bound form. The bleomycin assay only detects "free" iron and not iron bound to specific iron binding and storage proteins such as transferrin, ferritin, and haemoglobin, or to enzymes such as catalase. ${ }^{16}$ Thus BDI is never detected in the plasma of healthy adults.

We conducted a prospective study of preterm infants to determine whether plasma BDI is increased after blood transfusion and whether it exists in the hazardous ferrous state, which is an essentially pro-oxidant form of iron. We also examined the redox status of AA, which may be the most important reductant in 
Table 1 Clinical characteristics of the preterm infants

\begin{tabular}{|c|c|c|c|c|c|c|c|c|c|}
\hline $\begin{array}{l}\text { Patient } \\
\text { number }\end{array}$ & Sex & $\begin{array}{l}\text { Gestational age } \\
\text { (weeks) }\end{array}$ & $\begin{array}{l}\text { Birth } \\
\text { weight (g) }\end{array}$ & $\begin{array}{l}\text { Apgar score } \\
\text { at } 5 \text { min }\end{array}$ & $\begin{array}{l}\text { Age at blood } \\
\text { transfusion } \\
\text { (days) }\end{array}$ & $\begin{array}{l}\text { Duration of } \\
\text { oxygen therapy } \\
\text { (days) }\end{array}$ & $\begin{array}{l}\text { Age at the } \\
\text { start of enteral } \\
\text { feeding (days) }\end{array}$ & $\begin{array}{l}\text { Age on reaching } \\
100 \mathrm{ml} / \mathrm{kg} / \text { day } \\
\text { feeding (days) }\end{array}$ & Chronic complications \\
\hline 1 & M & 27 & 1076 & 9 & 25 & 3 & 5 & 29 & ROP stage $1^{\star}$ \\
\hline 2 & M & 27 & 1021 & 8 & 25 & 42 & 6 & 14 & CLD \\
\hline 3 & M & 30 & 1318 & 9 & 79 & 56 & 2 & 19 & CLD, ROP stage 3 \\
\hline 4 & $\mathrm{~F}$ & 26 & 717 & 2 & 32 & 58 & 7 & 21 & CLD, ROP stage 2 \\
\hline 5 & $\mathrm{~F}$ & 32 & 743 & 6 & 77 & 4 & 3 & 11 & ROP stage 2 \\
\hline 6 & M & 27 & 1070 & 8 & 38 & 63 & 9 & 33 & CLD, ROP stage 2 \\
\hline 7 & $\mathrm{~F}$ & 31 & 972 & 7 & 52 & 1 & 4 & 10 & None \\
\hline 8 & M & 29 & 1168 & 7 & 32 & 37 & 10 & 13 & None \\
\hline 9 & M & 31 & 1644 & 7 & 56 & 9 & 3 & 6 & None \\
\hline 10 & $M$ & 27 & 1220 & 5 & 38 & 51 & 7 & 13 & CLD, ROP stage 3 \\
\hline 11 & M & 26 & 932 & 9 & 20 & 61 & 1 & 21 & CLD, ROP stage 2 \\
\hline 12 & M & 28 & 1244 & 9 & 46 & 10 & 7 & 13 & None \\
\hline 13 & $\mathrm{~F}$ & 32 & 1150 & 7 & 35 & 10 & 4 & 14 & None \\
\hline 14 & $\mathrm{~F}$ & 26 & 1132 & 9 & 31 & 64 & 5 & 19 & ROP stage 2 \\
\hline 15 & M & 27 & 929 & 7 & 19 & 36 & 3 & 9 & CLD, ROP stage 2 \\
\hline 16 & $\mathrm{~F}$ & 32 & 608 & 3 & 14 & 120 & 9 & 101 & CLD, ROP stage 3 \\
\hline 17 & $\mathrm{~F}$ & 24 & 742 & 8 & 29 & 84 & 4 & 33 & CLD, ROP stage 3 \\
\hline 18 & M & 25 & 800 & 6 & 25 & 162 & 5 & 40 & CLD, ROP stage 4 \\
\hline 19 & $\mathrm{~F}$ & 27 & 1050 & 5 & 13 & 58 & 2 & 22 & ROP stage 1 \\
\hline 20 & $M$ & 26 & 995 & 3 & 43 & 52 & 5 & 14 & CLD, ROP stage 1 \\
\hline 21 & $\mathrm{M}$ & 25 & 730 & 6 & 42 & 83 & 3 & 35 & CLD, ROP stage 3 \\
\hline Median & & 27 & 1021 & 7 & 32 & 52 & 5 & 19 & \\
\hline
\end{tabular}

${ }^{\star}$ The highest stage in either eye.

ROP, Retinopathy of prematurity; CLD, chronic lung disease.

human plasma capable of reducing plasma BDI to its ferrous state, and plasma ferroxidase activity, which converts ferrous iron to ferric iron which can be incorporated into transferrin. We also performed these same studies in full term infants.

\section{Materials and methods}

This study was designed prospectively and the study protocol was approved by the ethics committee of our college hospital.

Investigations were performed after informed consent was obtained from the parents of the subjects.

SUBJECTS

The inclusion criteria for preterm infants were: (a) a gestational age $<32$ weeks; (b) absence of congenital heart disease, multiple malformations, or documented chromosomal abnormality; (c) availability of two serial plasma samples obtained just before and after blood transfusion. Between April 1996 and August 2000, 30 infants of gestational age $<32$ weeks received a blood transfusion in the neonatal intensive care unit of Osaka Medical College Hospital, and 21 of them fulfilled the above criteria. Table 1 summarises their clinical characteristics. CLD was defined as the requirement for extra oxygen supplementation beyond 28 days of life associated with symptoms of persistent respiratory distress and hazy lung fields on chest radiographs. ${ }^{18}$ ROP was classified according to the International Classification of Retinopathy of Prematurity. ${ }^{19}$ The arterial oxygen tension was maintained at $60-80 \mathrm{~mm} \mathrm{Hg}$, with monitoring by a transcutaneous oxygen monitor. All infants were fed their own mother's milk and our preterm formula containing 1.5 $\mathrm{mg} / \mathrm{dl}$ iron ( $50 \%$ each). None of the infants received therapeutic iron supplementation.

We also examined the effects of blood transfusion on iron status in full term infants. Within the study period, six full term infants of $>37$ weeks gestation received a blood transfusion at a median age of 29 days (range 14-64; not significantly different from the age at blood transfusion for preterm infants). The median gestational age and birth weight were 39 weeks (range 38-40) and $3073 \mathrm{~g}$ (range 2298-3282) respectively. The reasons for blood transfusion were rhesus haemolytic disease in two infants, gastrointestinal bleeding because of vitamin $\mathrm{K}$ deficiency in one, intraoperative haemorrhage in two (cystic hygroma and hydrocephalus), and early neonatal anaemia following abruptio placentae in one. In addition, we examined 10 healthy adult male volunteers with a median age of 35 years (range 26-44) as adult controls to obtain values before blood transfusion.

BLOOD TRANSFUSION AND SAMPLE COLLECTION In preterm infants, the haemoglobin level fell to below $12 \mathrm{~g} / \mathrm{dl}$ within a few weeks of birth, and subcutaneous injection of recombinant human erythropoietin was started at a median age of 22.5 days after birth (range 3-49) at a daily dose of $200 \mathrm{U} / \mathrm{kg}$ twice a week. The indications for blood transfusion were haemoglobin $<8 \mathrm{~g} / \mathrm{dl}$, haemoglobin $<10 \mathrm{~g} / \mathrm{dl}$ with ventilator support, or haemoglobin $<10 \mathrm{~g} / \mathrm{dl}$ with clinical evidence of cardiorespiratory problems (recurrent apnoea, tachypnoea, or tachycardia) lethargy, feeding difficulties, and/or poor weight gain. The blood used for transfusion was concentrated red blood cells provided by the Nihon Red Cross Society, which were stored at a packed cell volume of about $65 \%$ in MAP solution ${ }^{20}$ (mannitol, adenine, phosphate, citrate, glucose, and $\mathrm{NaCl}$ ) and were irradiated with $25 \mathrm{~Gy}$. Each infant received $10 \mathrm{ml} / \mathrm{kg}$ replacement red blood cells through a 24 gauge intravenous catheter at an infusion speed of $2 \mathrm{ml} / \mathrm{kg} / \mathrm{h}$. A preliminary study showed that the BDI concentration changed after $24 \mathrm{~h}$ and the change persisted for up to $72 \mathrm{~h}$ after blood transfusion. Therefore all measurements were carried out within $24 \mathrm{~h}$ before and 36-48 h after blood transfusion. Heparinised blood samples of volume $1.5 \mathrm{ml}$ were collected and immediately centrifuged $(1000 \mathrm{~g}, 10 \mathrm{~min})$, after which the plasma was 
Table 2 Plasma non-transferrin bound iron before and after blood transfusion (BT) in preterm and full term infants

\begin{tabular}{|c|c|c|c|c|}
\hline \multirow{2}{*}{$\begin{array}{l}\text { Patient } \\
\text { number }\end{array}$} & \multicolumn{2}{|c|}{ Bleomycin detectable iron $(\mu M)$} & \multicolumn{2}{|c|}{ Ferrous iron activity $(\mu M)$} \\
\hline & Before BT & After BT & Before BT & After BT \\
\hline \multicolumn{5}{|c|}{ Preterm infants } \\
\hline 1 & 2.5 & 5.6 & ND & ND \\
\hline 2 & 0 & 1.7 & 45.0 & 52.2 \\
\hline 3 & 0 & 3.8 & 21.0 & 16.7 \\
\hline 4 & 0 & 2.6 & ND & ND \\
\hline 5 & 0 & 2.1 & 59.0 & 62.3 \\
\hline 6 & 0 & 0 & 7.9 & 9.5 \\
\hline 7 & 0 & 0.2 & 0 & 4.7 \\
\hline 8 & 0 & 0 & 2.8 & 5.6 \\
\hline 9 & 0 & 0 & 2.9 & 6.3 \\
\hline 10 & 0 & 0 & 19.2 & 14.0 \\
\hline 11 & 1.1 & 0.8 & 4.9 & 11.6 \\
\hline 12 & 0 & 5.6 & 7.3 & 8.5 \\
\hline 13 & 0.3 & 2.3 & 21.6 & 30.4 \\
\hline 14 & 0 & 0.6 & 46.0 & 51.8 \\
\hline 15 & 2.2 & 3.1 & 30.2 & 31.4 \\
\hline 16 & 2.5 & 6.3 & 6.1 & 8.7 \\
\hline 17 & 4.5 & 20.7 & 11.2 & 59.7 \\
\hline 18 & 0 & 1.7 & 8.9 & 1.5 \\
\hline 19 & 0 & 0.8 & 12.2 & 14.4 \\
\hline 20 & 0 & 4.2 & 2.9 & 26.0 \\
\hline 21 & 3.4 & 3.5 & 67.6 & 80.4 \\
\hline Median & 0.0 & $2.1 \dagger$ & 11.2 & 14.4 \\
\hline$p^{\star}$ & & 0.005 & & 0.016 \\
\hline \multicolumn{5}{|c|}{ Full term infants } \\
\hline 1 & 0 & 0 & 10.2 & 8.2 \\
\hline 2 & 0 & 0 & 14.4 & 10.6 \\
\hline 3 & 0 & 0 & 0.3 & 0.2 \\
\hline 4 & 0 & 0.2 & 7.5 & 11.7 \\
\hline 5 & 0 & 0 & 2.8 & 6.5 \\
\hline 6 & 0 & 0 & 0.2 & 6.3 \\
\hline Median & 0 & 0 & 5.2 & 7.4 \\
\hline $\mathrm{p}^{\star}$ & & & & 0.463 \\
\hline
\end{tabular}

*Values obtained before and after transfusion were compared by the Wilcoxon signed rank test. tp $<0.05 v$ full term infants after transfusion (Mann-Whitney U test).

ND, Not done. $37^{\circ} \mathrm{C}$, and the thiobarbituric acid (TBA) test was then carried out. The absorbance of the resulting chromogen at $532 \mathrm{~nm}$ was measured spectrophotometrically, and the iron concentration was determined by comparison with a standard calibration curve created using an iron atomic absorption standard. To cancel out the effects of TBA reactive material other than DNA in the reaction mixture, a control without bleomycin was prepared for each sample. As a high concentration of AA was added to both the sample and standard mixtures to reduce ferric to ferrous iron and also to inhibit the ferroxidase activity of caeruloplasmin, the assay measured the sum of both ferrous and ferric iron.

BLEOMYCIN ASSAY FOR FERROUS IRON

Ferrous iron was measured by a slight modification of the BDI assay described above. ${ }^{21}$ The major difference was elimination of the reduction step. In addition, sensitive spectrofluorimetry was necessary to measure TBA reactive substances because there was less severe DNA damage. In contrast with the original method for the detection of ferrous iron described by Gutteridge, ${ }^{21}$ sodium azide (an inhibitor of ferroxidase) was not added so as to allow the detection of naturally occurring ferrous iron. Because this assay did not use AA or other iron reductants, and DNA degradation by bleomycin has an absolute requirement for ferrous iron, trace amounts of iron contaminating the standard, which may exist as ferric iron because of the pronounced instability of ferrous iron in aerobic solution, were not detectable. Accordingly, this assay could not directly quantify ferrous iron and instead detected "ferrous iron activity". In this report, the "activity" is expressed as millimolar ferrous iron relative to the standard calibration curve. Ferrous iron could not be assayed in two of the 21 transfusions (patients 1 and 4), because insufficient blood was available.

\section{FERROXIDASE ACTIVITY}

Plasma ferroxidase activity was measured as described previously. ${ }^{22}$ Because of limited sample volumes, ferroxidase activity could not be measured in patient 8 .

OTHER MEASUREMENTS

Plasma transferrin and caeruloplasmin levels were measured by nephelometry, ferritin was iron chelated by bleomycin to the ferrous stat The mixture was incubated for one hour at

Table 3 Plasma iron status before and after blood transfusion in preterm and full term infants

\begin{tabular}{|c|c|c|c|c|}
\hline & Total iron (mM) & Transferrin (mg/dl) & $\begin{array}{l}\text { Transferrin saturation } \\
(\%)\end{array}$ & Ferritin (mg/l) \\
\hline \multicolumn{5}{|l|}{ Preterm infants $(n=21)$} \\
\hline Before & $18.6(3.3-37.8) \dagger$ & $130.0(34.0-271.0) \dagger$ & $51.4(28.3-84.3)$ & $190.0(73.0-440.0)$ \\
\hline After & $18.3(4.8-38.0)$ & $116.0(31.0-260.0)$ & $54.1(25.4-78.3)$ & $220.0(78.0-320.0)$ \\
\hline Changes & $-1.0(-11.8-8.7)$ & $-3.0(-27.0-43.0)$ & $0.9(-16.9-21.7)$ & $12.0(-120.0-120.0)$ \\
\hline $\mathrm{p}$ Value ${ }^{\star}$ & 0.768 & 0.218 & 0.639 & 0.212 \\
\hline \multicolumn{5}{|l|}{ Full term infants $(n=6)$} \\
\hline Before & $14.8(11.3-26.6) \dagger$ & $161.0(156.0-174.0) \dagger$ & $34.8(24.6-61.0)$ & $255.0(120.0-490.0)$ \\
\hline After & $21.4(11.8-29.0)$ & $169.0(149.0-204.0)$ & $44.6(28.2-63.3)$ & $180.0(110.0-540.0)$ \\
\hline Changes & $0.1(-5.2-13.3)$ & $13.0(-20.0-34.0)$ & $0.7(-7.8-20.0)$ & $0.0(-30.0-50.0)$ \\
\hline $\mathrm{p}$ Value ${ }^{\star}$ & 0.500 & 0.345 & 0.500 & 0.655 \\
\hline Adult controls $(n=10)$ & $26.3(16.5-38.3)$ & $224.0(191.0-311.0)$ & $41.7(25.9-56.2)$ & $130.0(49.0-790.0)$ \\
\hline
\end{tabular}

Data are expressed as the median (range). The changes during blood transfusion were calculated by subtracting the values before transfusion from those after.

*Values obtained before and after transfusion were compared by the Wilcoxon signed rank test.

tp $<0.05 v$ adult controls by Mann-Whitney $U$ test according to Kruskal-Wallis test. 
Table 4 Redox status of plasma ascorbic acid before and after blood transfusion in preterm and full term infants

\begin{tabular}{|c|c|c|c|}
\hline & $A A(\mu M)$ & $D H A A(\mu M)$ & $D H A A \mid A A(\%)$ \\
\hline \multicolumn{4}{|c|}{ Preterm infants $(n=21)$} \\
\hline Before & $56.4(13.3-197.8)$ & $1.3(0.0-17.5)$ & $2.8(0.0-11.3)$ \\
\hline After & $44.6(12.7-191.5)$ & $2.6(0.0-26.0)$ & $6.0(0.0-24.7)$ \\
\hline Changes & $-2.5(-39.1-70.0)$ & $1.0(-0.2-19.6)$ & $3.5(-0.7-21.9)$ \\
\hline p Value ${ }^{\star}$ & 0.042 & 0.010 & 0.005 \\
\hline \multicolumn{4}{|c|}{ Full term infants $(n=6)$} \\
\hline Before & $57.4(40.5-70.8)$ & $2.3(1.9-4.9)$ & $4.6(3.6-8.6)$ \\
\hline After & $57.0(27.7-70.6)$ & $4.3(2.0-6.8)$ & $9.7(3.2-11.6)$ \\
\hline Changes & $-4.8(-12.8-25.7)$ & $1.5(-2.9-4.8)$ & $3.9(-3.7-6.9)$ \\
\hline p Value ${ }^{\star}$ & 0.463 & 0.208 & 0.116 \\
\hline
\end{tabular}

Data are expressed as the median (range). The changes during blood transfusion were calculated by subtracting the values before transfusion from those after.

$\star$ Values obtained before and after transfusion were compared by the Wilcoxon signed rank test. AA, Ascorbic acid; DHAA, dehydroascorbic acid.

measured by radioimmunoassay, and total iron was measured by the nitroso-PAPS method. ${ }^{22 a}$ Plasma AA and DHAA levels were determined as previously described. ${ }^{23}$

STATISTICAL ANALYSIS

Values before and after blood transfusion were compared using the Wilcoxon signed rank test. Intergroup differences between two groups were assessed by the Mann-Whitney $U$ test. Differences between three groups before blood transfusion were initially compared using the Kruskal-Wallis test, and, if a significant difference was found among the groups, comparison of the various pairs of groups was performed with the Mann-Whitney U test. A p $<0.05$ was considered to indicate significance. Data were analysed using SPSS for Macintosh, version 6.1 (SPSS Inc, Chicago, Illinois, USA).

\section{Results}

As shown in table 1, the median gestational age and birth weight of the preterm infants were 27 weeks and $1021 \mathrm{~g}$ respectively. About three quarters of the infants had at least one of such chronic complications as CLD or ROP. Oral feeding did not progress smoothly in most, and the median age at which milk intake reached $100 \mathrm{ml} / \mathrm{kg} /$ day was 19 days. Blood transfusion was carried out at a median age of 32 days, being performed after 1 month of life in 13 of the 21 transfusions.

Table 2 shows the non-transferrin bound free iron status. In preterm infants, total BDI was detected in $30 \%$ of the samples before blood transfusion, and BDI positive samples increased to $80 \%$ after transfusion. The median BDI concentration after transfusion was significantly higher than before. Ferrous iron was detected in all but one (patient 7) measurable sample, even before blood transfusion. Because the blank value in the ferrous iron assay is virtually zero, ferrous iron activity was high compared with the total BDI value. The median ferrous iron activity was also significantly higher after blood transfusion than before. In the full term infants, total BDI was not detected even after blood transfusion, except in one sample (patient 4). Ferrous iron activity was detected in all samples from full term infants, but the median value was only about half of that in preterm infants. In contrast with preterm infants, ferrous iron activity showed no changes after blood transfusion.

Table 3 shows the effects of blood transfusion on the plasma iron status, as estimated from commonly used variables. None of the values differed significantly after transfusion in both preterm and full term infants. The total iron and transferrin concentrations were significantly lower in preterm and full term infants than in adults, but transferrin saturation was comparable in the three groups. None of the comparisons between preterm and full term infants showed a statistically significant difference.

Table 4 shows changes in the AA redox status. After blood transfusion in preterm infants, the plasma AA concentration was significantly decreased, whereas the DHAA level and the DHAA/AA ratio were significantly increased compared with before the transfusion. Unlike preterm infants, the AA redox status of full term infants did not change after blood transfusion.

As shown in table 5, the plasma ferroxidase activity in preterm infants was only about one third of that in adults. Full term infants also had a lower ferroxidase activity than adults, but it was over twofold higher than in preterm infants. The caeruloplasmin levels of preterm infants were significantly different from those of full term infants or healthy adults, whereas there were no significant differences between full term infants and the adult controls. The AA concentration in preterm and full term infants was higher than that in adults, but the difference was not statistically significant. The plasma ratio of AA to caeruloplasmin in the three groups was in inverse order to the ferroxidase activity. The median ratio in preterm infants was fourfold higher than that in adult controls and was also significantly higher than in full term infants. The difference in the ratio of AA to caeruloplasmin between full term infants and adult controls was only about half as large, but it was still significant.

In addition, plasma samples obtained from 10 healthy adult volunteers contained neither BDI nor ferrous iron. Neither type of nontransferrin bound iron was detected in the

Table 5 Plasma ferroxidase activity and related variables

\begin{tabular}{lllll}
\hline & $\begin{array}{l}\text { Ferroxidase activity } \\
(\text { IU/l) }\end{array}$ & $\begin{array}{l}\text { Caeruloplasmin } \\
(\mu M)\end{array}$ & $\begin{array}{l}\text { AA } \\
(\mu M)\end{array}$ & $\begin{array}{l}\text { AA/caeruloplasmin } \\
\text { (molar ratio) }\end{array}$ \\
\hline Preterm infants $(\mathrm{n}=20)$ & $259.0(68.0-603.0)^{\star} \dagger$ & $0.7(0.3-1.9)^{\star} \dagger$ & $56.6(13.3-197.8)$ & $84.7(9.9-188.9)^{\star} \dagger$ \\
Full term infants $(\mathrm{n}=6)$ & $587.5(504.0-624.0)^{\star}$ & $1.6(1.3-2.0)$ & $57.4(40.5-70.8)$ & $34.8(30.2-39.3)^{\star}$ \\
Adult controls $(\mathrm{n}=10)$ & $704.0(640.0-792.0)$ & $1.8(1.5-2.0)$ & $38.5(14.0-61.0)$ & $21.9(7.8-38.9)$
\end{tabular}

All values were from samples obtained just before blood transfusion and are expressed as the median (range).

${ }^{\star} \mathrm{p}<0.05 v$ adult controls by the Mann-Whitney U test according to the Kruskal-Wallis test.

tp $<0.05 v$ full term infants by the Mann-Whitney U test according to the Kruskal-Wallis test.

$\mathrm{AA}=$ ascorbic acid 
MAP blood used for transfusion (data not shown).

\section{Discussion}

The usual explanation for the close association of blood transfusion with ROP is that it involves increased oxygen delivery to the retina because of the relatively low oxygen affinity of adult haemoglobin compared with fetal haemoglobin. However, this explanation is not consistent with the finding that exchange transfusion is not always related to the development of ROP. ${ }^{1}$ Apart from increased oxygen delivery, the role of iron has also attracted attention because preterm infants may suffer from iron overload after blood transfusion. ${ }^{24-27}$ This speculation is supported by the report that serum ferritin increases after transfusion in preterm infants. ${ }^{28}$ Accordingly, the possibility that loosely bound free iron may act as a catalyst in the formation of reactive oxygen species has been proposed as a theoretical basis for the association of blood transfusion with ROP.

In this study, we have shown that blood transfusion caused a significant increase in the plasma non-transferrin bound free iron level in preterm infants. We also found that seven of the 21 preterm infants had BDI even before blood transfusion, which is consistent with other reports. ${ }^{41329}$ Surprisingly, BDI was detectable despite the existence of reserve iron binding capacity provided by transferrin that was not fully iron saturated. However, this has already been reported in some preterm infants and in asphyxiated term infants. ${ }^{133031} \mathrm{An}$ in vitro study showed that BDI became detectable in the presence of transferrin with over $80 \%$ saturation when iron in the form of ferrous ammonium sulphate was added to plasma from a healthy adult male, ${ }^{32}$ but the origin of the BDI is still not known.

We found that BDI was partly in the reduced ferrous form in preterm infants. Ferrous iron is thought to be an essentially pro-oxidant form of iron, and the presence of ferrous iron activity has been reported in cord plasma from preterm and full term babies. ${ }^{33}$ Before binding to transferrin, iron must be converted into the ferric state by ferroxidase. The ferroxidase activity in our preterm infants was considerably reduced to about one third of the adult value. In human plasma, the main component of ferroxidase activity is caeruloplasmin, ${ }^{34}$ and the plasma concentration of caeruloplasmin in our preterm infants was less than half of that in adults. Furthermore, the ferroxidase activity of caeruloplasmin is known to be inhibited by high levels of AA, which reduces ferric iron to ferrous iron in competition with ferroxidase. ${ }^{35}$ An in vitro study showed that the ferroxidase activity of caeruloplasmin began to decline when the AA/caeruloplasmin ratio was about $100-200 .^{35}$ In this study, the median molar $\mathrm{AA} /$ caeruloplasmin ratio in the preterm infants was 84.7 , which was about fourfold higher than in adults. Thus the existence of plasma iron in the reduced ferrous state may be related to a decrease in ferroxidase activity resulting from the net effect of low caeruloplasmin and high

\section{Key messages}

- Plasma non-transferrin bound iron was significantly increased in preterm infants after blood transfusion and existed partly in the ferrous pro-oxidant form

- The increase in plasma non-transferrin bound iron after blood transfusion was not observed in full term infants

AA levels in preterm infants. The raised plasma DHAA level and the increased DHAA/AA ratio together with the reduced AA concentration after blood transfusion suggested a possible role for AA in the reduction of ferric iron to ferrous iron, although it is also possible that the increase in DHAA may have resulted from the oxidation of AA acting as a sacrificial antioxidant to scavenge free radicals.

In full term infants, unlike preterm infants, BDI was not detected even after blood transfusion. Ferrous iron activity was already present before transfusion, but did not increase after. The ferroxidase activity of full term infants was more than double that in preterm infants, as expected from the fact that full term infants had a higher caeruloplasmin and similar AA level, resulting in a lower $\mathrm{AA} /$ caeruloplasmin ratio than preterm infants. However, the ferroxidase activity in full term infants was insufficient to eliminate ferrous iron activity because of the extremely high sensitivity of the assay compared with that for BDI. To eliminate ferrous iron activity completely, ferroxidase activity should reach the adult level.

So far, it has not been confirmed that non-transferrin bound iron is in fact capable of catalysing the formation of reactive oxygen species in vivo. In this study, most of the preterm infants developed ROP and/or CLD. Although either complication may already have occurred before blood transfusion in our cases, it is still possible that transfusion accelerated the progression of these conditions. Recently, free radicals have gained attention as redox signalling molecules as well as the classical highly reactive villains that indiscriminately destroy biological components. ${ }^{36}$ Both ROP and CLD have the common pathological feature of proliferative fibrotic changes, and free radicals are known to play a prominent role in the transduction of proliferative stimuli. ${ }^{37}$ Therefore, in addition to the possibility that tissue damage through BDI catalysed free radical reactions may trigger the progression of ROP or CLD, it is also possible that only a slight increase in free iron after blood transfusion promotes redox signalling, which leads to proliferation of these conditions.

In conclusion, we have shown that plasma non-transferrin bound iron is significantly increased after blood transfusion in preterm infants and that some of it exists in the ferrous form, possibly because of a considerable decrease in ferroxidase activity. This finding was specific for preterm infants and was never observed in full term infants. Accordingly, BDI catalysed generation of reactive oxygen species 
may be responsible for the clinical association of blood transfusion with ROP or CLD.

We thank Drs $M$ Mino, A Nagai, $\mathrm{H}$ Moji, $\mathrm{H}$ Tanaka, $\mathrm{H}$ Kamegai, Y Seiki, and Y Suzuki for their assistance and advice, Kamegai, Y Seiki, and Y Suzuki for their assistance and advice, and M Imanishi, M Kobayashi, and H Ikeuchi for excellent laboratory assistance. We are also grateful to E Hata and the nursing staff of the neonatal intensive care unit for their help in sample collection. This work was supported by Grant-in-Aids (00211128) for Scientific Research from the Ministry of
Education, Science, and Culture, Japan and a Grant for Pediatric Research (8C-2) from the Ministry of Health and Welfare, Japan.

1 Sacks LM, Schaffer DB, Anday EK, et al. Retrolental fibroplasia and blood transfusion in very low-birth-weigh infants. Pediatrics 1981;68:770-4.

2 Clark C, Gibbs JAH, Maniello R, et al. Blood transfusion: a possible risk factor in retrolental fibroplasia. Acta Paediat Scand 1981;70:535-9.

3 Shohat M, Reisner SH, Krikler R, et al. Retinopathy of prematurity: incidence and risk factors Pediatrics 1983;72:159-63.

4 Cooke RWI, Drury JA, Yoxall CW, et al. Blood transfusion and chronic lung disease in preterm infants. Eur F Pediatr 1997; 156:47-50.

5 Silvers KM, Gibson AT, Russell JM, et al. Antioxidant activity, packed cell transfusions, and outcome in premature
infants. Arch Dis Child Fetal Neonatal Ed 1998;78:F214infa.

6 Bard H, Widness JA. The life span of erythrocytes transfused to preterm infants. Pediatr Res 1997;42:9-11.

7 Saugstad OD. Oxygen toxicity in the neonatal period. Acto Paediatr Scand 1990;79:881-92.

8 Ogihara T, Okamoto R, Kim HS, et al. New evidence for the involvement of oxygen radicals in triggering neonatal chronic lung disease. Pediatr Res 1996;39:117-19.

9 Ogihara T, Hirano K, Morinobu T, et al. Raised concentrations of aldehyde lipid peroxidation products in premature infants with chronic lung disease. Arch Dis Child Fetal Neonatal Ed 1999;80:F21-5.

10 Ogihara T, Kitagawa M, Miki M, et al. Susceptibility of neonatal lipoproteins to oxidative stress. Pediatr Res 1991;29:39-45.

11 Gopinathan V, Miller NJ, Milner AD, et al. Bilirubin and ascorbate antioxidant activity in neonatal plasma. FEBS Lett 1994;349:197-200.

12 Ripalda MJ, Rudolph N, Wong SL Developmental patterns of antioxidant defense mechanisms in human erythroof antioxidant defense mechan

13 Evans PJ, Evans R, Kovar IZ, et al. Bleomycin-detectable iron in the plasma of premature and full-term neonates. FEBS Lett 1992:303:210-12.

14 Halliwell B, Gutteridge JMC. Role of free radicals and catalytic metal ions in human disease: an overview. Methods Enzymol 1990;186:1-85.

15 Halliwell B. Vitamin C: antioxidant or pro-oxidant in vivo? Free Radic Res 1996;25:439-54.

16 Gutteridge JMC, Rowley DA, Halliwell B. Superoxidedependent formation of hydroxyl radicals in the presence of iron salts: detection of 'free' iron in biological systems by using bleomycin-dependent degradation of DNA. Biochem f 1981;199:263-5.

17 Gutteridge JMC, Hou Y. Iron complexes and their reactivity in the bleomycin assay for radical-promoting looselyin the bleomycin assay for radical-promoting lo
bound iron. Free Radic Res Commun 1986;2:143-51.
18 Ogawa Y, Fujimura M, Goto A, et al. Epidemiology of neonatal chronic lung disease in Japan. Acta Paediatr fpn 1992;34:663-7.

19 Commitee for the classification of retinopathy of prematurity. An international classification of retinopathy of prematurity. Pediatrics 1984;74:127-33.

20 Sasakawa S, Shiba T, Mura T, et al. Development of additive solution MAP for storage of red cell concentrates. Fapanese fournal of Transfusion Medicine 1991;37:398-403.

21 Gutteridge JMC. Ferrous ions detected in cerebrospinal fluid by using bleomycin and DNA damage. Clin Sci 1992;82:315-20.

22 Johnson DA, Osaki S, Frieden E. A micromethod for determination of ferroxidase (ceruloplasmin) in human plasma. Clin Chem 1967;13:142-50.

22a MakinoT, Kiyonaga M, Kina K. A sensitive, direct colorimetric assay of serum iron using the chromogen, nitro-PAPS. Clin Chim Acta 1988;171:19-28.

23 Ogihara T, Kim HS, Hirano K, et al. Oxidation products of uric acid and ascorbic acid in preterm infants with chronic lung disease. Biol Neonate 1998;73:24-33.

24 Sullivan JL. Iron, plasma antioxidants, and the 'oxygen radical disease of prematurity'. $A m$ f Dis Child 1988;142:1341-4

25 Sullivan JL. Retinopathy of prematurity: low iron binding capacity may contribute. BMF 1993;307:1353.

26 Hesse L, Eberl W, Schlaud M, et al. Blood transfusion: iron load and retinothy of prematurity. Eur $\mathcal{f}$ Pediatr 1997;156:465-70.

27 Inder TE, Clemett RS, Austin NC, et al. High iron status in very low birth weight infants is associated with an increased risk of retinopathy of prematurity. $f$ Pediatr 1997;131:541-4.

28 Shaw JCL. Iron absorption by the preterm infant. The effect of transfusion and iron supplements on the serum ferritin of transfusion and iron supplements on the serum
levels. Acta Paediatr Scand 1982;299(suppl):83-9.

29 Kime R, Gibson A, Yong W, et al. Chromatographic method for the determination of non-transferrin-bound iron suitable for use on the plasma and bronchoalveolar lavage fluid of preterm babies. Clin Sci 1996;91:633-8.

30 Dorrepaal CA, Berger HM, Benders MJNL, et al. Nonprotein-bound iron in postasphyxial reperfusion injury of the newborn. Pediatrics 1996;98:883-9.

31 Van Bel F, Shadid M, Moison RMW, et al. Effect of allopurinol on postasphyxial free radical formation, cerebral hemodynamics, and electrical brain activity. Pediatrics hemodynamics, a

32 Berger TM, Polidori MC, Dabbagh A, et al. Antioxidant activity of vitamin $\mathrm{C}$ in iron-overloaded human plasma. $\mathcal{F}$ Biol Chem 1997;272:15656-60.

33 Berger HM, Mumby S, Gutteridge JMC. Ferrous ions detected in iron-overloaded cord blood plasma from preterm and term babies: implications for oxidative stress. Free Radic Res 1995;22:555-9.

34 Gutteridge JMC, Stocks J. Caeruloplasmin: physiological and pathological perspectives. CRC Crit Rev Clin Lab Med and pathological p

35 Gutteridge JMC. Plasma ascorbate levels and inhibition of the antioxidant activity of caeruloplasmin. Clin Sci 1991;81:413-17.

36 Suzuki JS, Forman HJ, Sevanian A. Oxidants as stimulators of signal transduction. Free Radic Biol Med 1997;22:26985.

37 Poli G, Parola M. Oxidative damage and fibrogenesis. Free Radic Biol Med 1997;22:287-305. 